\section{Overriding safety measures}

Viruses are always striving to outwit their host, and staying one step ahead is proving difficult in our fight against HIV-1. The expression of inhibitors, known as restriction factors, by humans and nonhuman primates provides an innate mechanism that confers resistance to infection with various retroviruses. However, it seems that HIV-1 can hijack a host protein to counteract the inhibition of virus replication mediated by these factors. Reporting in Nature Medicine, Towers et al. show that the interaction between HIV-1 capsid protein (CA) and the host cell protein cyclophilin A (CYPA) modulates the sensitivity of HIV-1 to host restriction factors.

The CYPA binding site in CA is positioned close to residues that are involved in recognition by restriction factors. So, the authors investigated how the interaction between CA and CYPA influences the sensitivity of HIV-1 to restriction factors. Disruption of the CYPA-CA interaction either by

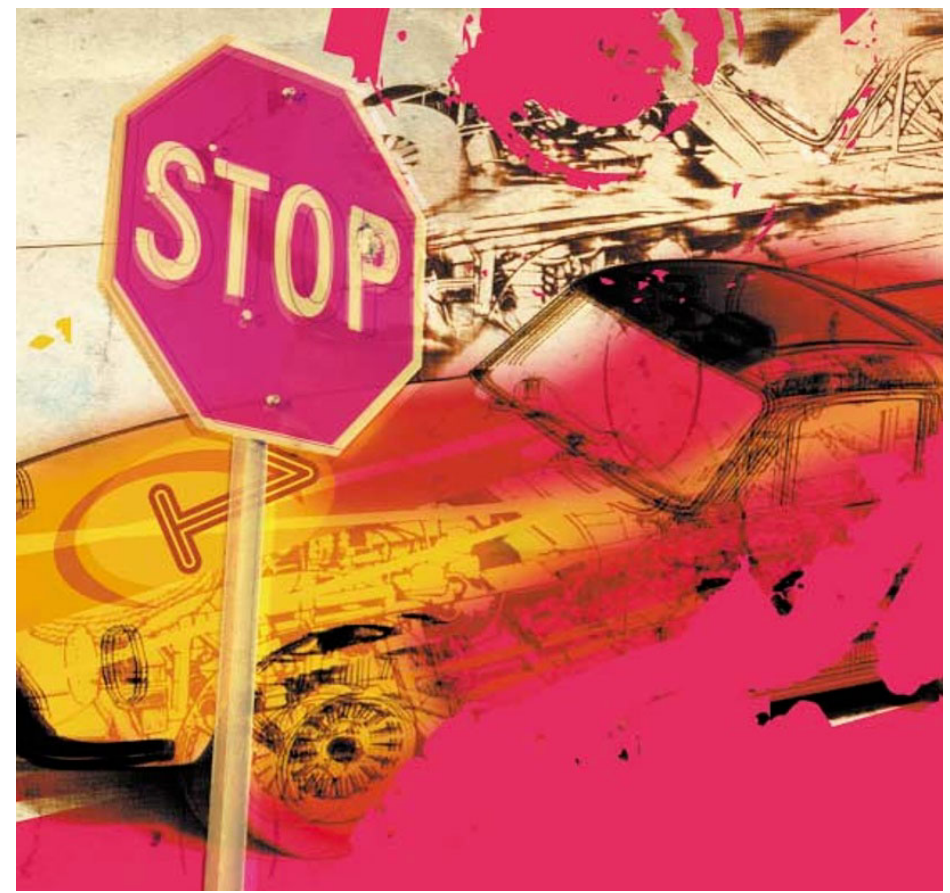

mutating the CYPA binding site in HIV-1 CA or by treating the target cells with cyclosporin A (CsA), which effectively competes for the binding of CYPA, had different effects on HIV-1 infectivity depending on the species of the reduced the infectivity of HIV-1, as HIV-1 became sensitive to the restriction factor REF1. By contrast, efficient infection of owl monkey (OMK) cells occurred when the CYPA-CA interaction was prevented, indicating that this binding event is required for restriction by OMK cells.

Therefore, it seems that specific adaptation of HIV-1 in human cells, but not unnatural hosts, has allowed HIV-1 to avoid recognition by restriction factors and override innate antiviral safety measures.

Lucy Bird

(1) References and links ORIGINAL RESEARCH PAPER Towers, G. J. et al. Cyclophilin A modulates the sensitivity of et al. Cyclophilin A modulates the sensitivity of
HIV-1 to host restriction factors. Nature Med. HIV-1 to host restriction factors. Nature target cell. In human cells, inhibition of the CYPA-CA interaction

\section{IN BRIEF}

\author{
ANTIBODY RESPONSES
}

AID mutant analyses indicate requirement for class-switch-specific cofactors.

Ta, V.-T. et al. Nature Immunol. 10 August 2003 (DOI: 10.1038/ni964)

Activation-induced cytidine deaminase (AID) is known to be the only B-cell-specific factor required for both class-switch recombination (CSR) and somatic hypermutation (SHM) during the maturation of an antibody response. How then does AID differentially regulate these two processes? Honjo and colleagues show that this might be achieved by specific cofactors that regulate substrate interaction. Point mutations in the carboxyterminal domain of AID abrogated CSR but did not affect SHM, which indicates the involvement of a CSR-specific cofactor that binds to the carboxyl terminus of AID.

\section{B-CELL DEVELOPMENT}

The nonimmunoglobulin portion of $\lambda 5$ mediates cell-autonomous pre-B cell receptor signaling.

Ohnishi, K. \& Melchers, F. Nature Immunol. 3 August 2003 (DOI: 10.1038/ni959)

Expression of the pre- $\mathrm{BCR}$ - composed of one rearranged heavy chain and a surrogate light chain (SLC; which is itself composed of VpreB and $\lambda 5$ ) - is a crucial intermediate step in B-cell development, allowing $B$ cells to proliferate in a ligand-independent manner. Deletion or mutation of the amino-terminal nonimmunoglobulin region of $\lambda 5$ led to increased cell-surface expression of the pre-BCR, but decreased pre-BCR aggregation and decreased phosphorylation of proteins in the pre-BCR complex. These results indicate that the amino-terminal region of $\lambda 5$ is required for signal transduction by the pre-BCR in response to receptor crosslinking. No ligands or other cell types were added to the pre- $\mathrm{B}$ cells, so the authors propose that this crosslinking involves a linking factor produced by the pre- $\mathrm{B}$ cell itself or non-covalent interactions between two $\lambda 5$ non-immunoglobulin regions.

\section{LYMPHOCYTE MIGRATION}

Cutting edge: the B cell chemokine CXC chemokine ligand 13/B lymphocyte chemoattractant is expressed in the high endothelial venules of lymph nodes and Peyer's patches and affects B cell trafficking across high endothelial venules.

Ebisuno, Y. et al. J. Immunol. 171, 1642-1646 (2003)

The chemokines CC-chemokine ligand 21 (CCL21) and CCL19 are localized to high endothelial venules (HEVs) and are involved in controlling T-cell trafficking to lymph nodes or Peyer's patches. In this study, Ebisuno et al. analysed the role of the B-cell chemoattractant CXCL13 in the trafficking of B cells across HEVs. CXCL13 was found to be expressed on the luminal surface of HEVs. B-cell arrest in HEVs of Peyer's patches was impaired in CXCL13deficient mice, but superfusion of the Peyer's patches with CXCL13 restored the expression of CXCL13 at the luminal surface and some $B$ cells were once again able to adhere. This study shows that CXCL13 expressed by HEVs has a role in the trafficking of B cells in lymphoid organs. 